NOTE

\title{
A New Method for Obtaining Local Ordering in Amorphous Polymers from X-Ray Scattering Intensity
}

\author{
Mikio NumaKaWA and Akira OdaJIMA \\ Department of Applied Physics, Faculty of Engineering, Hokkaido University, \\ Nishi 8-chome, Kita 13-jo, Kita-ku, Sapporo 060, Japan.
}

(Received November 13, 1980)

\begin{abstract}
KEY WORDS X-Ray Scattering / Modelled Radial Distribution Function / trans Fraction / Local Order / Amorphous Polyethylene /
\end{abstract}

The radial distribution function (RDF) analysis of polymers in the amorphous state can be used to indicate local order and molecular chain packing. The chain conformation is characterized by unconditional probabilities, $P_{t}$ for trans and $P_{g}$ for gauche, and/or higher-order probabilities, for instance, $P_{t t}$ and $P_{t g}$, which are defined by a set of rotational angles about the skeletal bonds.

Since polyethylene (PE) is one of the simplest molecules in polymers, a number of RDF studies on its amorphous structure have been reported in the last few years. ${ }^{1-5}$ Nevertheless, the local conformation of the PE chain in the amorphous state has not been quantitatively evaluated by these RDF studies. Recently, we made a very careful RDF analysis of amorphotized PE and ethylenepropylene random copolymer in order to obtain the trans fraction in the chains. ${ }^{6}$

On the other hand, Windle and his coworkers ${ }^{7}$ proposed a method for evaluating the trans fraction by comparing the experimental scattering curve with those calculated for various structure models and showed that any significant degree of long sequences of regular conformation cannot be found in molten PE.

We have shown that the experimental RDF of amorphous PE can be modelled for the interchain damped oscillation in terms of a set of simple functions. ${ }^{8}$ In this paper, we propose a new method for obtaining information on the trans fraction and the interchain packing in amorphous PE by a comparison of the experimental scattering curve and the Fourier transform of the RDF modelled for the intrachain and interchain orders.

\section{METHOD}

Figure 1a shows the $s$-weighted normalized intensity, si(s), of the amorphotized PE reported by Yoda et al. ${ }^{4}$, where $s=4 \pi \sin \theta / \lambda . \mathrm{Cu}-K \alpha_{1}$ rays were monochromatized by $\alpha$-quarz on the incident side, and X-ray diffraction measurement was carried out at room temperature. PE was amorphotized by $\gamma$ ray irradiation of $4000 \mathrm{Mrad}$ (4000 Mrad PE).

The experimental RDF, $G_{\mathrm{e}}(r)$, is derived from the inverse Fourier transform of $s i(s) \cdot \exp \left(-\gamma s^{2}\right)$ as,

$$
G_{\mathrm{e}}(r)=2 r / \pi \int_{s_{\text {min }}}^{s_{\max }} s i(s) \cdot \exp \left(-\gamma s^{2}\right) \sin (r s) \mathrm{d} s
$$

where, $\exp \left(-\gamma s^{2}\right)$ is a damping factor to minimize the termination errors due to the finite range of $s$ from $s_{\min }\left(=0.1 \AA^{-1}\right)$ to $s_{\min }\left(=7.7 \AA^{-1}\right)$. Figure $1 \mathrm{~b}$ shows $s i(s) \cdot \exp \left(-\gamma s^{2}\right)$, in which $\exp \left(-\gamma s^{2}\right)$ takes a value of 0.2 at $s_{\max }=7.7 \AA^{-1}$. The dotted line in Figure 2 indicates $G_{\mathrm{e}}(r)$ above $4 \mathrm{~A}$ of the radial distance, which is obtained from $s i(s) \cdot \exp \left(-\gamma s^{2}\right)$ shown in Figure 1b. The sharp peaks are smeared out in $G_{\mathrm{e}}(r)$ because of the finite $s$ range and the damping factor. ${ }^{4}$

The modelled RDF, $G_{\mathrm{m}}(r)$, in the range of $r$ smaller than $4 \AA$ is formed as follows: (1) We chose $r_{1}$ at which $G_{\mathrm{m}}\left(r_{1}\right)$ takes a minimum value. For $0<r<r_{1}$, we have,

$$
G_{\mathrm{m}}^{(1)}(r)=-4 \pi r^{2} \bar{\rho}+G_{\mathrm{p}}^{(1)}(r)
$$




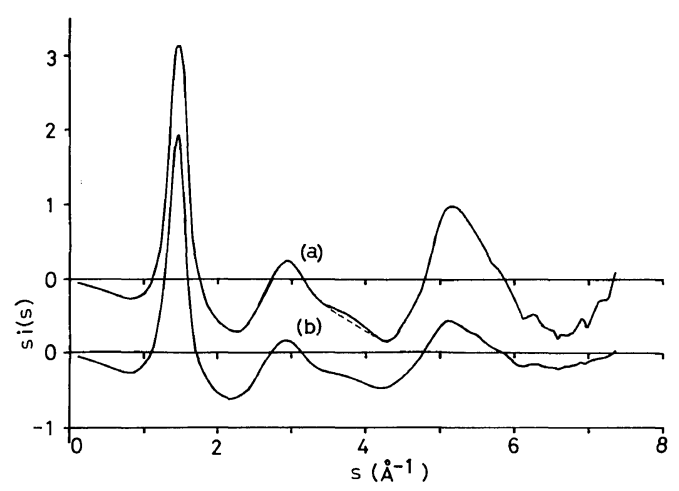

Figure 1. Experimental $s i(s) \cdot \exp \left(-\gamma s^{2}\right)$ for PE amorphotized by $\gamma$-ray irradiation of 4000 Mrad. Damping factor $\exp \left(-\gamma s^{2}\right)$ equals 1.0 for (a) and takes a value of 0.2 at $s_{\max }=7.7 \AA^{-1}$ for (b).

where $\bar{\rho}\left(=0.041 \AA^{-3}\right)$ is the average number of $\mathrm{C}$ atoms per unit volume corresponding to the macroscopic density $\left(=0.95 \mathrm{~g} \mathrm{~cm}^{-3}\right)$ of the $4000 \mathrm{Mrad}$ $\mathrm{PE}$ and $G_{\mathrm{p}}^{(1)}(r)$ expresses the intrachain peaks within $r_{1}$. (2) We took $r_{2}$ near 4A in which $G_{\mathrm{e}}(r)=0$.

For $r_{1}<r<r_{2}$, we have,

$$
G_{\mathrm{m}}^{(2)}(r)=Q \cdot r\left(r_{2}-r\right)+G_{\mathrm{p}}^{(2)}(r)
$$

where $G_{\mathrm{p}}^{(2)}(r)$ expresses intrachain peaks between $r_{1}$ and $r_{2}$ and $Q$ is a parameter. The value of $Q$ is determined from the condition which must be satisfied by $G_{\mathbf{m}}(r)^{9}$ :

$\int_{0}^{\infty} G_{\mathrm{m}}(r) \mathrm{d} r=-\left\{Z_{\mathrm{C}}^{2}+(2-x) Z_{\mathrm{H}}^{2}\right\} /\left\{Z_{\mathrm{C}}+(2-x) Z_{\mathrm{H}}\right\}^{2}$

where $Z_{\mathrm{C}}$ and $Z_{\mathrm{H}}$ are the atomic numbers of the $\mathrm{C}$ and $\mathrm{H}$ atoms, and $2-x$ is the composition ratio of the $\mathrm{H}$ atoms to the $\mathrm{C}$ atoms, that is, the $\mathrm{H} / \mathrm{C}$ ratio, where $x$ denotes the fraction of $\mathrm{H}$ atoms liberated by $\gamma$-ray irradiation. Elementary analysis showed $2-x=1.79$ for $4000 \mathrm{Mrad}$ PE. The resultant function, $G_{\mathrm{p}}(r)\left(=G_{\mathrm{p}}^{(1)}(r)+G_{\mathrm{p}}^{(2)}(r)\right)$ for the intrachain $\mathrm{C}-\mathrm{C}$ and $\mathrm{C}-\mathrm{H}$ peaks within $r_{2}$ is expressed by the following form ${ }^{10}$ :

$$
\begin{aligned}
G_{\mathrm{p}}(r)= & 2 r / \pi \int_{0}^{\infty}\left\{f_{\mathrm{C}}+(2-x) f_{\mathrm{H}}\right\}^{-2} \sin (r s) \\
& \times \mathrm{d} s\left[f _ { \mathrm { C } } { } ^ { 2 } X ^ { \mathrm { CC } } \left\{\sum_{i=1}^{2} \sin \left(r_{i}^{\mathrm{CC}}\right) / r_{i}^{\mathrm{CC}}\right.\right.
\end{aligned}
$$

$$
\begin{aligned}
& \left.+\sum_{n=1}^{2} P_{n} \sin \left(r_{3 n}^{\mathrm{CC}} s\right) / r_{3 n}^{\mathrm{CC}}\right\} \\
& +f_{\mathrm{C}} f_{\mathrm{H}}\left\{\sum_{i=1}^{2} X_{i}^{\mathrm{CH}} \sin \left(r_{i}^{\mathrm{CH}} s\right) / r_{i}^{\mathrm{CH}}\right. \\
& \left.\left.+\sum_{n=1}^{3} X_{3}^{\mathrm{CH}} P_{n} \sin \left(r_{3 n}^{\mathrm{CH}} s\right) / r_{3 n}^{\mathrm{CH}}\right\}\right]
\end{aligned}
$$

Here, $f_{\mathrm{C}}$ and $f_{\mathrm{H}}$ are the atomic scattering factors for $\mathrm{C}$ and $\mathrm{H}$ atoms, $r_{i}^{\mathrm{CC}}$ (or $r_{i}^{\mathrm{CH}}$ ) is the $\mathrm{C} \cdots \mathrm{C}$ (or $\mathrm{C} \cdots \mathrm{H}$ ) distance separated by $i$ bonds, and $X_{i}^{\mathrm{CC}}$ (or $\left.X_{i}^{\mathrm{CH}}\right)$ the number of equivalent pairs. $r_{3 n}^{\mathrm{CC}}$ and $r_{3 n}^{\mathrm{CH}}$ are the $\mathrm{C} \cdots \mathrm{C}$ and $\mathrm{C} \cdots \mathrm{H}$ distances for $i=3$, where $n=1$ denotes trans and $n=2$ and 3 gauche, and $P_{n}$, the fraction of the $n$-state. These distances are shown in Table I. The $r_{i}^{\mathrm{CC}}$ and $r_{i}^{\mathrm{CH}}$ distances for $i=1$ and 2 are independent of the chain conformation.

On the other hand, for the long distance $r>r_{2}$ where the interchain contribution is dominant, a modelled RDF is determined that will fit the experimental RDF, $G^{\mathrm{e}}(r)$, shown by the dotted line in Figure 2.

For $r>r_{2}$, we have,

Table I. Intrachain distances between atoms separated by $i$ bonds

\begin{tabular}{cccc}
\hline$i$ & $n$ & $r^{\mathrm{Cc}}(\mathrm{A})$ & $r^{\mathrm{CH}}(\mathrm{A})$ \\
\hline \multirow{2}{*}{1} & & 1.54 & 1.09 \\
2 & & 2.54 & 2.16 \\
& 1 (trans) & 3.9 & 2.77 \\
3 & 2 (gauche) & 3.0 & 2.77 \\
& 3 ( & & 3.48 \\
\hline
\end{tabular}

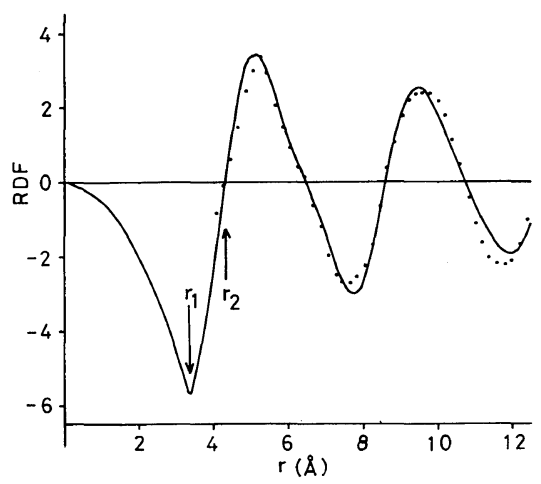

Figure 2. Modelled RDF $G_{\mathrm{m}}(r)$ (solid line) and experimental RDF $G_{\mathrm{e}}(r)$ (dotted line) for PE amorphotized 


$$
\begin{aligned}
G_{\mathrm{m}}^{(3)}(r)= & A \cdot r \cdot \sin w\left(r-r_{2}\right) \cdot \exp \left\{-\left(r-r_{2}\right) / a\right\} \\
& +B \cdot r \cdot \sin 2 w\left(r-r_{2}\right) \cdot \exp \left\{-\left(r-r_{2}\right) / b\right\}
\end{aligned}
$$

where the second term in the $G_{\mathrm{m}}^{(3)}(r)$ expresses the asymmetry of the damped oscillation appearing in $G_{\mathrm{e}}(r)$ for $r>r_{2} . A, B, w, a$ and $b$ are adjustable paremeters determined so that $G_{\mathrm{m}}^{(3)}(r)$ fits $G_{\mathrm{e}}(r)$ and $A=0.72, B=0.22, w=1.43, a=5$ and $b=3$ were chosen. Although intrachain contibutions are included in $G_{\mathrm{m}}^{(3)}(r)$, they are less dominant in the region of $r>r_{2}$ than interchain contributions.

\section{RESULTS AND DISCUSSION}

The solid line in Figure 2 shows the modelled RDF, $G_{\mathrm{m}}(r)\left(=G_{\mathrm{m}}^{(1)}(r)+G_{\mathrm{m}}^{(2)}(r)+G_{\mathrm{m}}^{(3)}(r)\right)$, in which the intrachain peaks, $G_{\mathrm{p}}(r)$, are omitted. There is good agreement between $G_{\mathrm{m}}^{(3)}(r)$ and $G_{\mathrm{e}}(r)$ over $4 \mathrm{~A}$. $s i(s)$ can be derived analytically from the Fourier transform of $G_{\mathrm{m}}(r)$ through eq 2-6 without numerical calculation. It is interesting to examine how the Fourier transforms of each part of the modelled RDF, $G_{\mathrm{m}}(r)$, contribute to $s i(s)$. Figures $3 \mathrm{a}, \mathrm{b}$ and $\mathrm{c}$ show the respective Fourier transforms $\left(s i^{(k)}(s)(k=1\right.$ to 3$\left.)\right)$ of $G_{\mathrm{m}}^{(1)}(r)+G_{\mathrm{m}}^{(2)}(r)-G_{\mathrm{p}}(r)$, the first and second terms in $G_{\mathrm{m}}^{(3)}(r)$. Figure 3a shows that the average number of $\mathrm{C}$ atoms per unit volume, $\bar{\rho}$ and the distances of $r_{1}$ and $r_{2}$ (parameters for the closest approach of atoms in the neighbouring chains) affect signifficantly $s i^{(1)}(s)$ up to

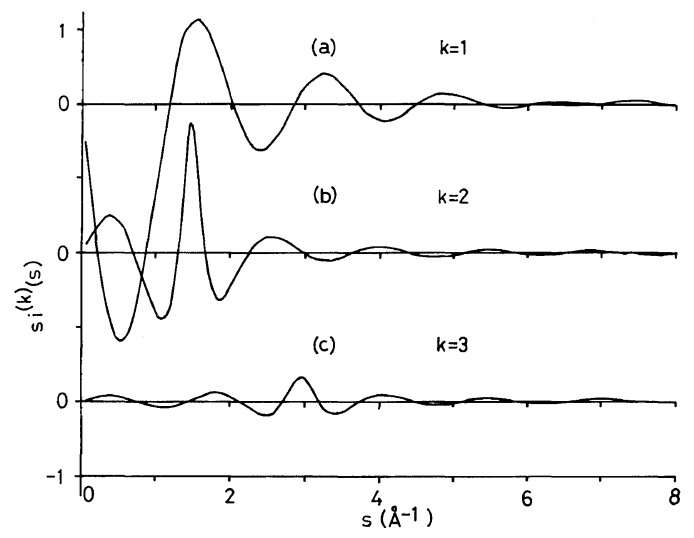

Figure 3. Fourier transforms $\left(s i^{(k)}(s)(k=1\right.$ to 3$\left.)\right)$ of (a) $G_{\mathrm{m}}^{(1)}(r)+G_{\mathrm{m}}^{(2)}(r)-G_{\mathrm{p}}(r)$, (b) the first term and (c) the second term in $G_{\mathrm{m}}^{(3)}(r) .\left(G_{\mathrm{m}}(r)=G_{\mathrm{m}}^{(1)}(r)+G_{\mathrm{m}}^{(2)}(r)+\right.$ $\left.G_{\mathrm{m}}^{(3)}(r)\right)$. $s=4 \AA^{-1}$. The heighest peak at $s=1.5 \AA^{-1}$ in the $s i^{(2)}(s)$ curve shown in Figure $3 \mathrm{~b}$ is due to the damped oscillation in $G_{\mathrm{m}}^{(3)}$ over $r_{2}$. On the other hand, the heighest peak at $s=3 \AA^{-1}$ shown in Figure $3 \mathrm{c}$ indicates that the second peak of the $s i(s)$ curve in Figure 1a strongly reflects the asymmetry in the radial distribution of interchain packing. Figures $4 \mathrm{a}, \mathrm{b}, \mathrm{c}$, and $\mathrm{d}$ show the Fourier transforms of $G_{\mathrm{p}}(r)\left(s i^{(4)}(s)\right)$ for the trans fractions $\left(P_{t}\right)$ of 1.0, $0.75,0.6$, and 0.45 , respectively. These curves show that the $s i(s)$ curve in the region of $s>4 \AA^{-1}$ is determined for the most part only by the intrachain $\mathrm{C}-\mathrm{C}$ and the $\mathrm{C}-\mathrm{H}$ peaks of distances separated by $i$ bonds ( $i=1,2$ and 3$)$.

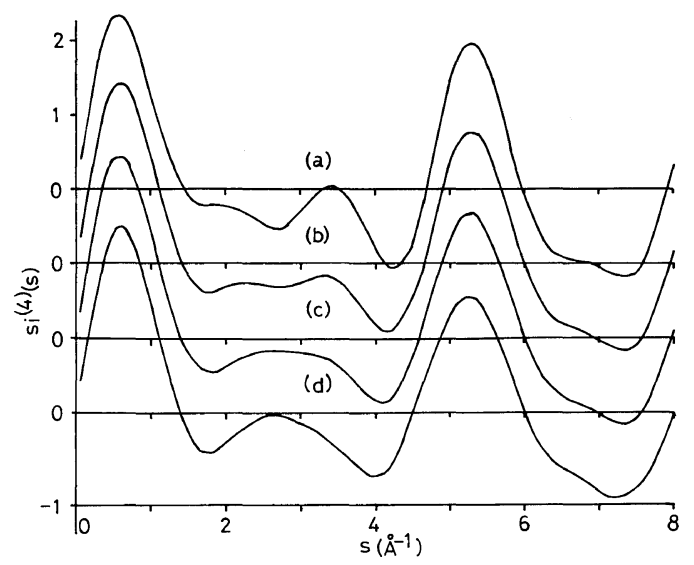

Figure 4. Fourier transform $\left(s i^{(4)}(s)\right)$ of intrachain peaks $G_{\mathrm{p}}(r)$ for trans fractions of (a) 1.0 , (b) 0.75 , (c) 0.6 , and (d) 0.45 .

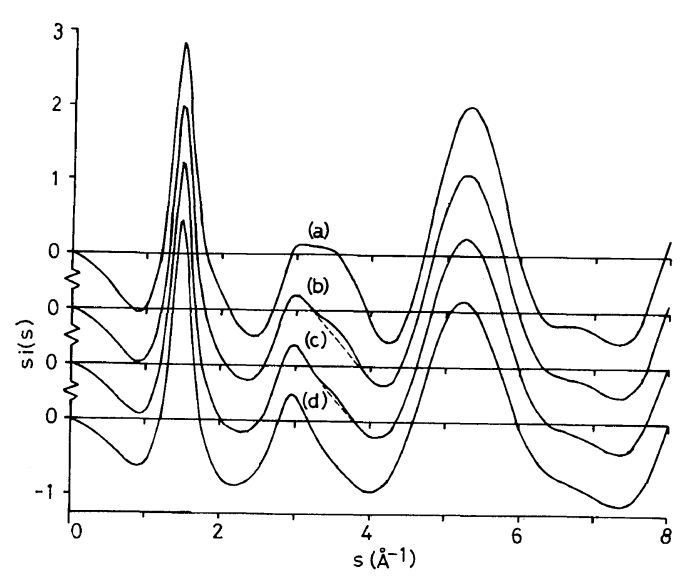

Figure 5. si $(s)$ curves calculated from $G_{\mathrm{m}}(r)$ for trans fractions of (a) 1.0 , (b) 0.75 , (c) 0.6 and (d) 0.45 . 
Figure 5 shows the si(s) curves sythesized from Figures 3 and 4 . It is noteworthy that the intensity of the shoulder appearing at $s \approx 3.5 \AA^{-1}$ is highest when $P_{t}=1.0$ and decreases as $P_{t}$ decreases. The area of the shoulder at $s \approx 3.5 \AA^{-1}$ separated by the dashed line in the experimental si(s) in Figure la is compared with those in the calculated $s i(s)$ as shown in Figure $5 b$ and $c$. The experimental area showed the best agreement with the calculated area for $P_{t}=0.65$. On the other hand, the trans fraction for PE $\gamma$-ray irradiated for $5000 \mathrm{Mrad}$ (5000 Mrad PE) was determined as 0.6 from our RDF study. ${ }^{6}$ The trans fraction of 0.65 for the $4000 \mathrm{Mrad} \mathrm{PE}$ is a little higher than 0.6 for the $5000 \mathrm{Mrad} \mathrm{PE}$. This suggests that regularity in the chain conformation was reduced with an increase in $\gamma$-ray irradiation from 4000 to 5000 Mrad, even though both the PE's were amorphotized. This may be related to the RDF result in which the regularity of interchain packing for $4000 \mathrm{Mrad} \mathrm{PE}$ is better than that for the 5000 Mrad. $^{4}$

The present method is very effective for studying the local ordering in polymers, provided a scattering intensity measured exactly within $5 \AA^{-1}$ of $s$ can be used. We are going to apply this method to the scattering curves for the $5000 \mathrm{Mrad} \mathrm{PE}$ and ethylene-propylene random copolymer, and their temperature dependence. Comparison between their chain conformations analyzed by this method and by the RDF method will be reported in the near future.
Acknowledgement. The authors wish to thank Dr. O. Yoda of Japan Atomic Energy Research Institute for providing X-ray scattering and RDF data on PE amorphotized by $\gamma$-ray irradiation of 4000 Mrad.

\section{REFERENCES}

1. I. Voigt-Martin and G. G. Mijlhoff, J. Appl. Phys., 46, 1165 (1975); ibid., 47, 3942 (1976).

2. G. W. Longman, G. D. Wignall, and R. P. Sheldon, Polymer, 17, 485 (1976); ibid., 20, 1063 (1979).

3. S. Kan and T. Seto, Rep. Prog. Polym. Phys. Jpn., 19, 219 (1976).

4. O. Yoda, I. Kuriyama, and A. Odajima, Appl. Phys. Lett., 32, 18 (1978); J. Appl. Phys., 49, 5468 (1978).

5. M. Gupta and G. S. Yeh, J. Macromol. Sci., Phys., B16(2), 225 (1978).

6. M. Numakawa and A. Odajima, Rep. Prog. Polym. Phys. Jpn., 23, 201 (1980).

7. R. Lovell, G. R. Mitchel, and A. H. Windle, Faraday Discuss, No. 68, 46 (1979); G. R. Mitchel, R. Lovell, and A. H. Windle, to be submitted.

8. M. Numakawa and A. Odajima, Rep. Prog. Polym. Jpn., 22, 141 (1979).

9. A. Gunier, "X-ray Diffraction in Crystals, Imperfect Crystals and Amorphous Bodies," W. H. Freeman and Company, San Francisco, 1963, p 71.

10. M. Numakawa and A. Odajima, Rep. Prog. Polym. Phys. Jpn., 21, 145 (1978). 